\title{
Intersubband Raman laser from GalnAs/AllnAs double quantum wells
}

\author{
Maxi Scheinert, ${ }^{\text {a) }}$ Hans Sigg, ${ }^{\text {b) }}$ and Soichiro Tsujino \\ Laboratory for Micro-and Nanotechnology, Paul-Scherrer-Institut, 5232 Villigen-PSI, Switzerland
}

Marcella Giovannini and Jérôme Faist

Physics Department, University of Neuchâtel, Rue A.L. Breguet 1, 2000 Neuchâtel, Switzerland

(Received 17 August 2007; accepted 8 September 2007; published online 26 September 2007)

\begin{abstract}
We demonstrate optically pumped GaInAs/AlInAs based intersubband lasers operating at a wavelength of $\sim 5.5 \mu \mathrm{m}$ applying a simple three-level design. Using a subnanosecond long pulsed excitation laser tunable over the linewidth of the transition from the first level to the third level $E_{13}$, Raman shift in the lasing spectra was observed. Lasing output power of $\sim 14 \mathrm{~W}$ and an internal conversion efficiency between pump and intersubband laser of $\sim 1.3 \%$ at $70 \mathrm{~K}$ is observed. (C) 2007
\end{abstract} American Institute of Physics. [DOI: 10.1063/1.2790781]

Since the first quantum cascade laser (QCL) based on intersubband optical transitions was demonstrated in $1994,{ }^{1}$ its physics and device applications have attracted wide interest. ${ }^{2,3}$ Within the past decade, also optically pumped intersubband lasers have been demonstrated. ${ }^{4}$ Compared to the electrically pumped cascade laser, optically pumped lasers are based on the simpler active layer design and waveguides with reduced losses due to the lack of electrical contacts. In this kind of system, the Raman lasing which has been observed in many materials, ${ }^{5-7}$ can be strongly enhanced by bringing excitation and lasing energy in resonance with electronic transitions. For a three-level coupled well, the intersubband transition $E_{13}$ from level $E 1$ to level $E 3$ is resonantly excited while the transition $E_{32}$ from $E_{3}$ to the second level $E_{2}$ defines the lasing wavelength. The transition $E_{21}$ from $E_{2}$ to $E_{1}$ defines the depopulation. Such an intersubband Raman laser was first demonstrated in a GaAs/AlGaAs material system at the lasing wavelength of $15 \mu \mathrm{m} .{ }^{8}$

In this letter, we demonstrate InP-based optically pumped intersubband lasers based on a three-level design in a double quantum well (DQW) which was grown on undoped (100 oriented) InP substrates by molecular beam epitaxy. Two kinds of GaInAs/AlInAs samples were investigated: Sample A was grown lattice matched while for samples B and C about $0.5 \%$ strain is introduced in order to achieve better confinement of the upper laser and to shift $E_{12}$. In the following discussion we will focus on samples $A$ and $B$ for lack of space. The layer sequence of the waveguide structure for both samples starting from the substrate is $0.6 \mu \mathrm{m}$ GaInAs cladding layer, $50 \mathrm{~nm}$ AlInAs core layer, the $1.1 \mu \mathrm{m}$ active region, a $50 \mathrm{~nm}$ AlInAs core layer, and a $1.1 \mu \mathrm{m}$ GaInAs cap layer. The active region consists of 60 periods of DQWs separated by a $9.5 \mathrm{~nm}$ thick AlInAs barrier. The center of this barrier is $n$ doped over $3 \mathrm{~nm}$ at 3 $\times 10^{17} \mathrm{~cm}^{-3}$. For sample A (sample B), each DQW is composed of $4.7 \mathrm{~nm}(5 \mathrm{~nm})$ GaInAs, $1.0 \mathrm{~nm}(1.2 \mathrm{~nm})$ AlInAs, and $3.5 \mathrm{~nm}(3.8 \mathrm{~nm})$ GaInAs. The corresponding band diagrams are shown in Fig. 1(a).

The design parameters were validated on the basis of absorption spectra taken at several temperatures $T$. Results of these measurements at 70 and $300 \mathrm{~K}$ are shown in Fig. 1(b)

\footnotetext{
${ }^{a)}$ Electronic mail: maxi.scheinert@psi.ch

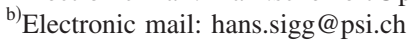

and summarized in Table I. For sample B, the $E_{12}$ transition could not be measured as it is below the cutoff of the mercury cadmium telluride (MCT) detector.

As pump laser, a $50 \mathrm{~mm}$ long periodically poled (PP) Li-niobate crystal is excited by $\sim 0.4$ ns long laser pulses obtained from a $Q$-switched neodymium-doped yttrium aluminum garnet (PowerChip, teemphotonics) laser operating at $1064 \mathrm{~nm}$ with a typical pulse energy of $70 \mu \mathrm{J}$ at $1 \mathrm{kHz}$ repetition rate. By parametric downconversion, infrared pulses at a wavelength around $3 \mu \mathrm{m}$ having a pulse energy of $\sim 1.7 \mu \mathrm{J}$ (measured after transport through the optic and the vacuum windows) are obtained. The wavelength is tuned by

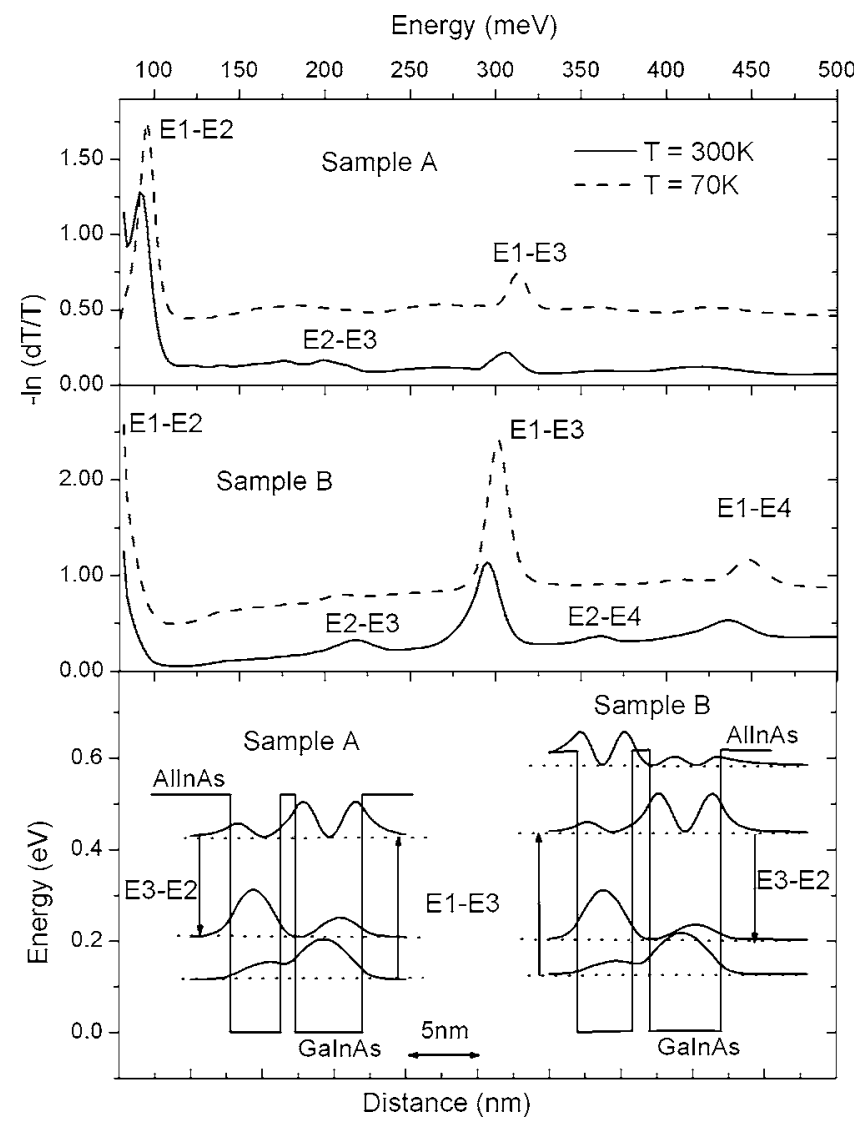

FIG. 1. (a) Measured absorption spectra for temperatures of 70 and $300 \mathrm{~K}$. The $70 \mathrm{~K}$ data are shifted for clarity. (b) Conduction band structure and square moduli of the wavefunctions for one period of each DQW structure. 
TABLE I. Transition energies, corresponding linewidth $\Gamma$, and peak absorption $\alpha_{\text {peak }}$ obtained in multipass $45^{\circ}$ waveguide geometry. Energies marked by $*$ and $* *$ are calculated from $E_{12}-E_{13}$ and $E_{23}-E_{13}$, respectively.

\begin{tabular}{cccccc}
\hline \hline Sample & $E_{13}$ & $\Gamma_{13}$ & $\alpha_{\text {peak }}$ & $E_{12}$ & $E_{23}$ \\
\hline$A(70 \mathrm{~K})$ & $313 \mathrm{meV}$ & $10 \mathrm{meV}$ & $0.55 \mathrm{~cm}^{-1}$ & $95 \mathrm{meV}$ & $217 \mathrm{meV}^{*}$ \\
$(300 \mathrm{~K})$ & $305 \mathrm{meV}$ & $14 \mathrm{meV}$ & $0.3 \mathrm{~cm}^{-1}$ & $92 \mathrm{meV}$ & $212 \mathrm{meV}$ \\
$B(70 \mathrm{~K})$ & $302 \mathrm{meV}$ & $13 \mathrm{meV}$ & $2.1 \mathrm{~cm}^{-1}$ & - & - \\
$(300 \mathrm{~K})$ & $295 \mathrm{meV}$ & $17 \mathrm{meV}$ & $1.3 \mathrm{~cm}^{-1}$ & $76 \mathrm{meV}^{* *}$ & $219 \mathrm{meV}$ \\
\hline \hline
\end{tabular}

setting the temperature of the crystal between 50 and $200{ }^{\circ} \mathrm{C}$ and by using PP elements with different periods. The light is coupled into the $\sim 200 \mu \mathrm{m}$ thick samples via a $45^{\circ}$ polished substrate facet (see inset of Fig. 2). The broad area cavity is formed by cleaved facets, and the length of the cavity is equal to $5 \mathrm{~mm}$. The samples were measured in a liquid helium optical cryostat equipped with $\mathrm{ZnSe}$ windows. For spectral analysis, the lasing light was detected by a midinfrared MCT detector located behind a grating spectrometer with a resolution of $0.2 \mathrm{meV}$ for a slit width of $400 \mu \mathrm{m}$. The responsitivity of the MCT detector has been calibrated using a pyroelectric detector ( $\mathrm{P} 3$, Molectron). To minimize the atmospheric absorption due to water and $\mathrm{CO}_{2}$, the spectrometer was nitrogen purged and the outcoupled lasing light was guided from the cryostat to the detector through a nitrogen flow box.

For excitation at resonance energy-pumping energy $\hbar \omega_{p}$ coincides with $E_{13}$-strong lasing with a total output pulse energy from a single facet up to $2 \mathrm{~nJ}$ is obtained at $70 \mathrm{~K}$ for sample A. The lasing energies $\hbar \omega_{l}$ are found to be $\sim 217 \mathrm{meV}(=5.71 \mu \mathrm{m})$ and $\sim 222 \mathrm{meV}(=5.59 \mu \mathrm{m})$ for samples A and B, respectively, which is in good agreement with energy values given in Table I. The internal lasing efficiency is evaluated for sample A by considering the losses from the window of the cryostat and the lenses in front of the detector of 0.95 and 0.8 , respectively. As we expect the same output on both facets, a total emitted pulse power of $5.3 \mathrm{~nJ}$ is achieved. On the incoupling side, the reflection losses of factor 0.3 and the absorption $1-\exp \left(-2 \alpha_{\text {peak }} L\right)=0.36$ have to be considered, where $L$ is the length of the cavity along

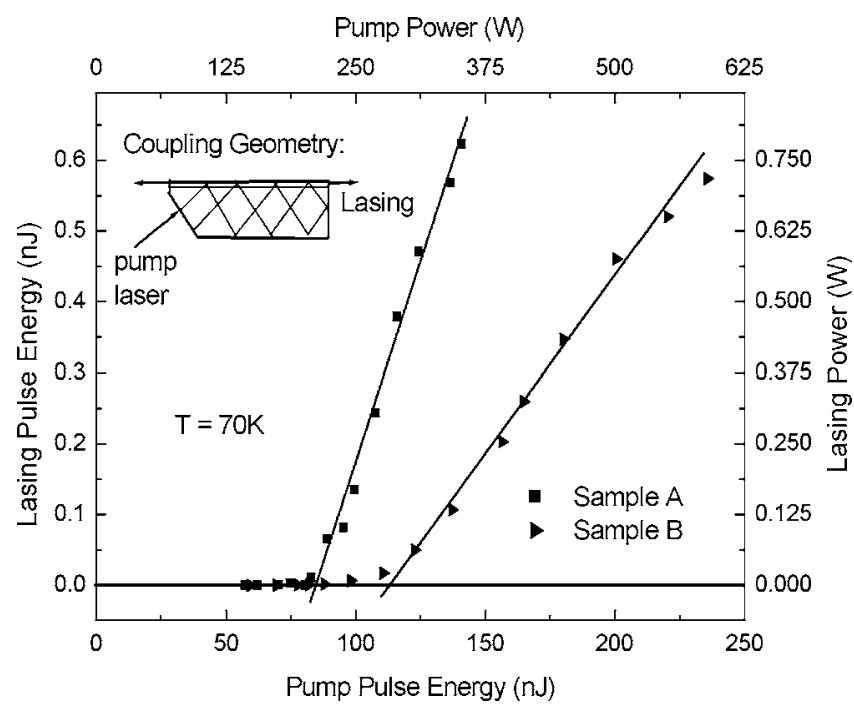

FIG. 2. Lasing threshold curves for both samples at $T=70 \mathrm{~K}$ for resonant excitation with a fit of the experimental data points for clarity. The inset shows the coupling geometry. which the excitation pulse is traveling twice, in forward and backward directions. Hence, the internal energy conversion efficiency is $1.3 \%$ which corresponds to a quantum efficiency of about $7 \%$.

Figure 2 shows the threshold characteristics for both samples for the pump photon energy $\hbar \omega_{p}$ equal to $E_{13}$ at $70 \mathrm{~K}$. The internal threshold pump pulse energy (absorbed power) of the lasing is equal to $86 \mathrm{~nJ}(208 \mathrm{~W})$ for sample A and $113 \mathrm{~nJ}(283 \mathrm{~W})$ for sample B. Based on the cavity length and a spot size of $\sim 100 \mu \mathrm{m}$ of the excitation laser which was determined in a separate experiment using a pinhole, the corresponding threshold intensities are equal to 52 and $57 \mathrm{~kW} / \mathrm{cm}^{2}$, respectively. The highest operation temperatures are found to be $\sim 190$ and $\sim 120 \mathrm{~K}$ for samples $\mathrm{A}$ and $\mathrm{B}$, respectively. To explain intersubband laser action in a coupled well, population inversion gain $g_{\mathrm{PI}}$ and Raman gain $g_{R}$ have been invoked. ${ }^{9}$ From the threshold measurements, we find a higher threshold pump power than for common III-V QCLs which evidences a Raman process. To further identify the nature of gain, we measured the dependence of the lasing energy $\hbar \omega_{l}$ on the pumping energy $\hbar \omega_{p}$. This relation is plotted for three samples at $80 \mathrm{~K}$ in Fig. 3 and for sample $\mathrm{A}$ the spectra over the whole tuning range are shown in the inset of Fig. 3. In all cases, a linear dependence with a near unity slope is found. The deviation in the low energy tail is assigned to inhomogeneities within the sample structure. Besides this, the measured offset $\left(\hbar \omega_{p}-\hbar \omega_{l}\right)$ corre-

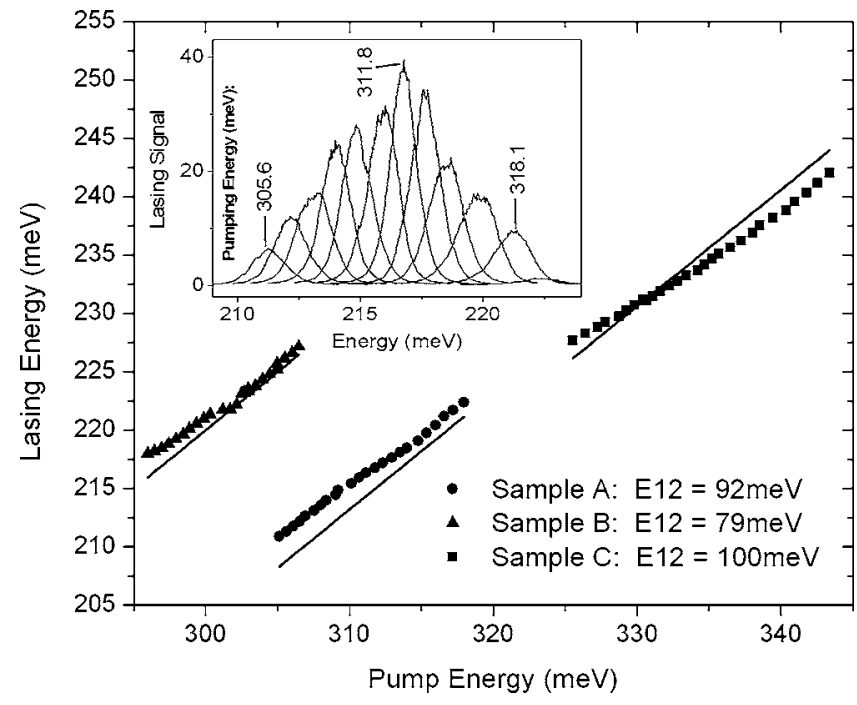

FIG. 3. Peak position of the emission spectra vs pumping energy for samples A-C (points). The solid lines are the shifts expected from the absorption measurements. Sample $\mathrm{C}$ was designed to have a depopulation energy $E_{12}$ higher than double phonon resonance $(\sim 100 \mathrm{meV})$ and is shown for completeness reasons but not discussed in further detail. For all samples, the shift of the peak emission with pumping wavelength follows the Raman shift. The inset shows the spectra of sample A over the whole tuning range. 


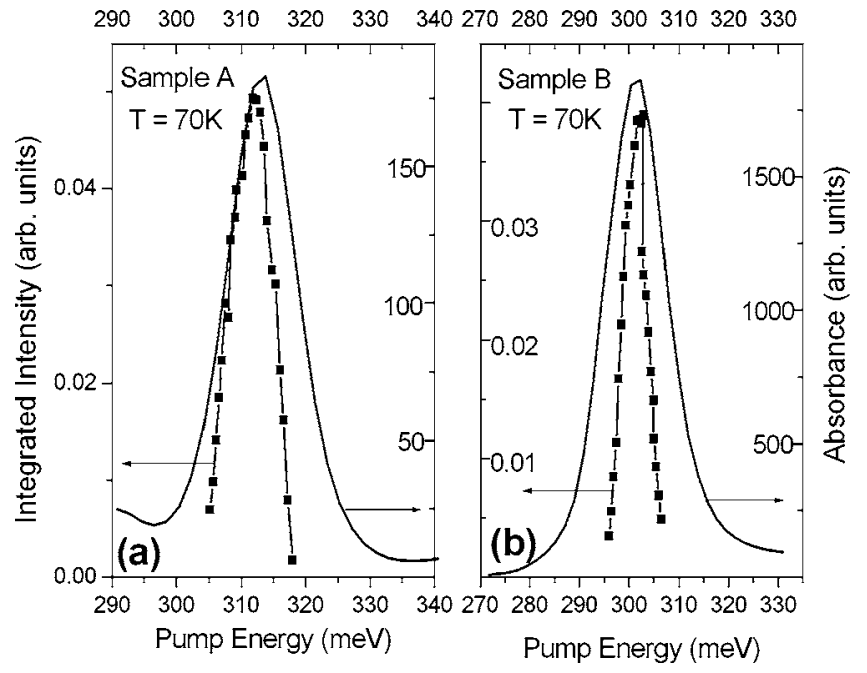

FIG. 4. Comparison between absorption and lasing tuning range for sample A (a) and sample B (b) at $T=70 \mathrm{~K}$. The lines with symbols are the integrated spectra for the corresponding pump energy. The lines give the measured absorption spectra of the $E_{13}$ transition.

sponds within $2 \mathrm{meV}$ to the experimentally determined $E_{12}$ transition. The fact that the value of $E_{12}$ is changed between 79,92 , and $100 \mathrm{meV}$ in samples $\mathrm{B}, \mathrm{A}$, and $\mathrm{C}$, respectively, and do not correspond to phonon energies demonstrates that the Raman gain has a pure electronic origin.

From the above discussion we can state that the Raman process is the dominating gain mechanism in our samples although lifetimes calculated at an in-plane wavevector of $k=0$ predict a favorable ratio of $\tau_{32} / \tau_{21}=3.5$ for the population inversion. A more careful look at the electron dynamics show that this simple picture is invalid: Electrons that leave the third subband at $k=0$ will scatter to the subbands $n=1$ and $n=2$ with a large kinetic energy and thus will be distributed in $k$ space. The intersubband lifetime $\tau_{21}$ from $E_{2}$ to $E_{1}$ at such large in-plane momentum is $1.6 \mathrm{ps}$ instead of $0.7 \mathrm{ps}$ at $k=0$. However, further simulations are necessary for a more detailed understanding of the $k$ dependence as also hot carrier effects are expected to be relevant to electron distribution at these strong pumping intensities. However, also from this simple consideration, we already expect only a marginal population inversion between the third and second subbands. This picture is supported by the plot of the inte- grated laser intensity versus measured absorption of the $E_{13}$ transition, as shown in Fig. 4: The maximum gain is in resonance with the $E_{13}$ absorption peak and the lasing can be tuned within the linewidth. Using Eq. (1) in Ref. 10, this behavior can only be modeled for a lower state lifetime only slightly smaller than the upper state lifetime, hence for a small population inversion gain and a clearly pronounced Raman gain.

In conclusion, we realized an optically pumped GaInAs/AlInAs lasers emitting at wavelengths of 5.5-5.7 $\mu \mathrm{m}$. By detuning the excitation wavelength, we have shown Raman lasing and we argue that this observation implies a marginal population inversion. This is also indicated by the relatively high threshold obtained for this structure. Finally, we note that this behavior offers a chance to realize a $\mathrm{Si} / \mathrm{SiGe}$ optically pumped laser as the lifetimes in that material system are short and heavily influenced by scattering mechanisms.

The authors acknowledge S. Stutz for device preparation and technical support. This work was financial supported by the Swiss National Science Foundation.

${ }^{1}$ J. Faist, F. Capasso, D. L. Sivco, A. L. Hutchinson, C. Sirtori, and A. Y. Cho, Science 264, 553 (1994).

${ }^{2}$ J. Faist, F. Capasso, C. Sirtori, D. L. Sivco, and A. Y. Cho, in Intersubband Transitions in Quantum Wells: Physics and Device Applications II, Semiconductors and Semimetals, edited by H. C. Liu and F. Capasso (Academic, San Diego, 2000), Vol. 62, Chap. 1, pp. 1-83.

${ }^{3} \mathrm{C}$. Sirtori and R. Teissier, in Intersubband Transitions in Quantum Structures, 1st ed., edited by R. Paiella (McGraw-Hill, New York, 2006), Chap. 1 , pp. 1-39.

${ }^{4}$ O. Gauthier-Lafaye, P. Boucaud, F. H. Julien, S. Sauvage, J. M. Lourtioz, V. Thierry-Mieg, and R. Planel, Appl. Phys. Lett. 71, 3619 (1997); O. Gauthier-Lafaye, F. H. Julien, S. Cabaret, J. M. Lourtioz, G. Strasser, E. Gornik, M. Helm, and P. Bois, ibid. 74, 1537 (1999).

${ }^{5}$ J. Nishizawa and K. Suto, J. Appl. Phys. 51, 2429 (1980).

${ }^{6}$ A. S. Grabtchikov, A. N. Kuzmin, V. A. Lisinetskii, V. A. Orlovich, G. I. Ryabtsev, and A. A. Demidovich, Appl. Phys. Lett. 75, 3742 (1999).

${ }^{7}$ H. Rong, R. Jones, A. Liu, O. Cohen, D. Hak, A. Fang, and M. Paniccia, Nature (London) 433, 725 (2005).

${ }^{8}$ H. C. Liu, I. W. Cheung, A. J. SpringThorpe, C. Dharma-waranda, Z. R. Wasilewski, D. J. Lockwood, and G. C. Aers, Appl. Phys. Lett. 78, 3580 (2001).

${ }^{9}$ J. B. Khurgin, G. Sun, L. R. Friedmann, and R. A. Soref, J. Appl. Phys. 78, 7398 (1995).

${ }^{10}$ M. Troccoli, A. Belyanin, F. Capasso, E. Cubukcu, D. L. Sivco and A. Y. Cho, Nature 433, 845 (2005). 